\title{
Is Ferroptosis a Future Direction in Exploring Cryptococcal Meningitis?
}

\author{
Xianbin $\mathrm{Xu}^{1 \dagger}$, Danfeng $\mathrm{Lin}^{2 \dagger}$, Sheng $\mathrm{Tu}^{1}$, Shiqi Gao ${ }^{3}$, Anwen Shao ${ }^{3 *}$ and Jifang Sheng ${ }^{1 *}$ \\ 1 State Key Laboratory for Diagnosis and Treatment of Infectious Diseases, Collaborative Innovation Center for Diagnosis and \\ Treatment of Infectious Diseases, The First Affiliated Hospital, College of Medicine, Zhejiang University, Hangzhou, China, \\ 2 Department of Surgical Oncology, Second Affiliated Hospital, School of Medicine, Zhejiang University, Hangzhou, China, \\ ${ }^{3}$ Department of Neurosurgery, Second Affiliated Hospital, School of Medicine, Zhejiang University, Hangzhou, China
}

\section{OPEN ACCESS}

Edited by: Stéphane Ranque,

Aix-Marseille Université, France

Reviewed by:

Peter R. Williamson,

National Institutes of Health $(\mathrm{NIH})$,

United States

Oscar Zaragoza,

Instituto de Salud Carlos III (ISCIII),

Spain

*Correspondence:

Jifang Sheng

jifang_sheng@zju.edu.cn

Anwen Shao

21118116@zju.edu.cn

${ }^{\dagger}$ These authors have contributed equally to this work

Specialty section:

This article was submitted to Microbial Immunology,

a section of the journal

Frontiers in Immunology

Received: 25 August 2020

Accepted: 03 March 2021

Published: 19 March 2021

Citation:

Xu X, Lin D, Tu S, Gao S, Shao A and

Sheng J (2021) Is Ferroptosis a

Future Direction in Exploring

Cryptococcal Meningitis?

Front. Immunol. 12:598601.

doi: 10.3389/fimmu.2021.598601
Cryptococcal meningitis $(\mathrm{CM})$ is the leading cause of mortality among patients infected with human immunodeficiency virus (HIV). Although treatment strategies for CM are continually being developed, the mortality rate is still high. Therefore, we need to explore more therapeutic strategies that are aimed at hindering its pathogenic mechanism. In the field of $\mathrm{CM}$, several studies have observed rapid iron accumulation and lipid peroxidation within the brain, all of which are hallmarks of ferroptosis, which is a type of programmed cell death that is characterized by iron dependence and lipid peroxidation. In recent years, many studies have confirmed the involvement of ferroptosis in many diseases, including infectious diseases such as Mycobacterium tuberculosis infection and coronavirus disease-2019 (COVID-19). Furthermore, ferroptosis is considered as immunogenic and pro-inflammatory as the ferroptotic cells release damage-associated molecular pattern molecules (DAMPs) and alarmin, both of which regulate immunity and pro-inflammatory activity. Hence, we hypothesize that there might be a relationship between this unique cell death modality and CM. Herein, we review the evidence of ferroptosis in CM and consider the hypothesis that ferroptotic cell death may be involved in the cell death of CM.

Keywords: cryptococcal meningitis, ferroptosis, iron accumulation, lipid peroxidation, immunomodulatory activity, inflammation, infection

\section{INTRODUCTION}

Cryptococcal meningitis (CM) is the most common lethal fungal infection in patients with acquired immune deficiency syndrome (AIDS). In 2009, Park et al. first estimated the global burden of HIVassociated cryptococcal infection, and identified approximately 957,900 new cases of CM per year worldwide, resulting in 624,700 deaths within three months after infection (1). Additional studies have also suggested an increasing number of annual global deaths, with CM being responsible for $15 \%$ of AIDS-related deaths $(2,3)$. A recent systematic review reported a total of 8,769 cases of cryptococcosis in mainland China from 1985 to 2010 through the use of CBMdisk (China Biology and Medicine data disc) database (4). However, the incidence of cryptococcosis in China is likely much higher than the reported number due to missed and misdiagnosed cases. According to the United Nations AIDS Program (UNAIDS), approximately 37.9 million people are currently living with HIV, and as HIV-infected patients have hypoimmunity, they are more prone to acquiring opportunistic infections, including CM. 
$\mathrm{CM}$ is a type of subacute meningoencephalitis that is mainly caused by Cryptococcus neoformans, which largely attacks patients with immunodeficiency, and leads to the development of fungal meningitis $(1,5,6)$. The main transmission route of cryptococcal infection is inhalation of yeast or basidiospores through the respiratory tract, though human-to-human or mother-to-child transmissions have been reported $(7,8)$. The main virulence factors of $C$. neoformans include production of melanin and polysaccharide capsule (9). Melanin is synthesized in C. neoformans by being primarily converted from catecholamines, such as dopa, dopamine, and epinephrine. As these catecholamines are neurotransmitters within the central nervous system (CNS) (10-12), C. neoformans have characteristics of neurotropism (13). Furthermore, several studies have demonstrated that $C$. neoformans is able to escape from attacks that are caused by the immune system by protecting melanin $(10,14)$. With regards to the polysaccharide capsule, it is able to exacerbate the toxicity of CM infection under some conditions, which include anti-phagocytosis, complement depletion, and inhibition of leukocyte migration (11, 12). As these are characteristics of $C$. neoformans, the most common presentation of invasive cryptococcal infection in patients living with HIV is lethal meningitis and meningoencephalitis.

Currently, the predominant therapies for HIV-associated CM include a combination of antifungal drugs, control of intracranial pressure, and appropriately timed initiation of highly active antiretroviral therapy (HAART) $(15,16)$. The treatment course involves an induction period, a consolidation period, and a maintenance period (16). Although novel treatment strategies for $\mathrm{CM}$ are continually being identified and developed, the mortality rate remains high due to a number of reasons, including infection of CNS, drug resistance, high cost, and availability of essential drugs. Therefore, there is a need to explore more treatment strategies and potential therapeutic targets. For instance, recent studies have shown that sertraline, a selective serotonin reuptake inhibitor antidepressant, exhibits excellent in vitro-in vivo antifungal activity (17-19). Some clinical trials have also identified that adjuvant sertraline can partially improve clearance of the cerebrospinal fluid (CSF) fungus (20-22). Unfortunately, a randomized, placebocontrolled, double-blind phase 3 trial confirmed that sertraline did not reduce the mortality of HIV-associated CM, which may be related to insufficient duration of therapeutic sertraline concentration (21). Moreover, it is important to keep exploring therapeutic strategies that are aimed at determining the pathogenesis of CM. Some recent studies have indicated increased iron accumulation and lipid peroxidation in CM, thus revealing a connection between $\mathrm{CM}$ and ferroptosis (23-27).

Recently, the role of ferroptosis in cell death has become a research hotspot and is associated with many diseases (28-30), such as tumors and ischemic organ injuries. Ferroptosis is known to play a role in various brain diseases (31-34), including stroke, Parkinson's disease (PD), and Alzheimer's disease (AD). There are also several studies that have shown the involvement of ferroptosis in infections caused by different pathogens, such as
Pseudomonas aeruginosa (35, 36), Mycobacterium tuberculosis (37-39), severe acute respiratory syndrome coronavirus 2 (SARS CoV-2) (40-42), and hepatitis B virus (HBV) $(43,44)$. Beyond that, ferroptosis is associated with immunogenic and proinflammatory activities. Ferroptotic cells are known to release DAMPs and immune-stimulating cellular components, which can be recognized by immune receptors, thus inducing cell death and inflammation. Furthermore, ferroptosis of immune cells during an infection can have an effect on immune function and create a beneficial environment for infectious agents. Taken together, ferroptosis plays a part in brain disease and infectious diseases, and likely regulates immunity and inflammation. Importantly, ferroptosis's key hallmarks, which include iron accumulation and lipid peroxidation, are known in CM. Therefore, we hypothesize that ferroptosis is involved in pathological process of $\mathrm{CM}$, and regulating ferroptosis represents a novel therapeutic target and strategy. Herein, determining what the function of ferroptosis is in CM and how ferroptosis affects the immune system in infectious diseases remains to be resolved.

\section{BRIEF HISTORY AND MECHANISMS OF FERROPTOSIS}

In 2003, Dolma et al. performed a synthetic lethal highthroughput screening to investigate the ability of 23,550 compounds to kill engineered tumorigenic cells, results of which showed that a compound named erastin was able to induce a non-apoptotic cell death process (45). Subsequently, in 2008, two small molecule compounds, Ras synthetic lethal 3 (RSL3) and Ras synthetic lethal 5 (RSL5), were observed to stimulate an iron-dependent non-apoptotic cell death, and shared similar properties to erastin (46). In 2012, Dixon et al. identified that erastin-induced oxidative iron-dependent cell death was morphologically different from the characteristics of apoptosis, necrosis, and autophagy. Furthermore, this particular form of cell death was named ferroptosis (28). In the following years, three different types of metabolism, iron metabolism, lipid metabolism, and amino acid metabolism, were described as the main mechanisms of ferroptosis (Figure 1) (28).

\section{Iron Metabolism}

Preliminary studies have identified that ferroptosis can be suppressed by co-treatment with iron chelators (deferoxamine), and potentiated by incubating with three different exogenous iron sources (28). These results support that iron metabolism is involved in pathogenesis of ferroptosis. Additionally, more in-depth research has validated the role of iron in ferroptosis (Figure 1). Extracellular $\mathrm{Fe}^{3+}$ bound to transferrin (Tf) are recognized by transferrin receptors (TFRs) on the cellular membrane, and imported into the cytoplasm (47). Next, $\mathrm{Fe}^{3+}$ is converted to $\mathrm{Fe}^{2+}$ in endosomes using a sixtransmembrane epithelial antigen of prostate 3 (STEAP3), a ferrireductase. Then, $\mathrm{Fe}^{2+}$ is released from the endosomes and into the cytoplasm through divalent metal transporter 1 


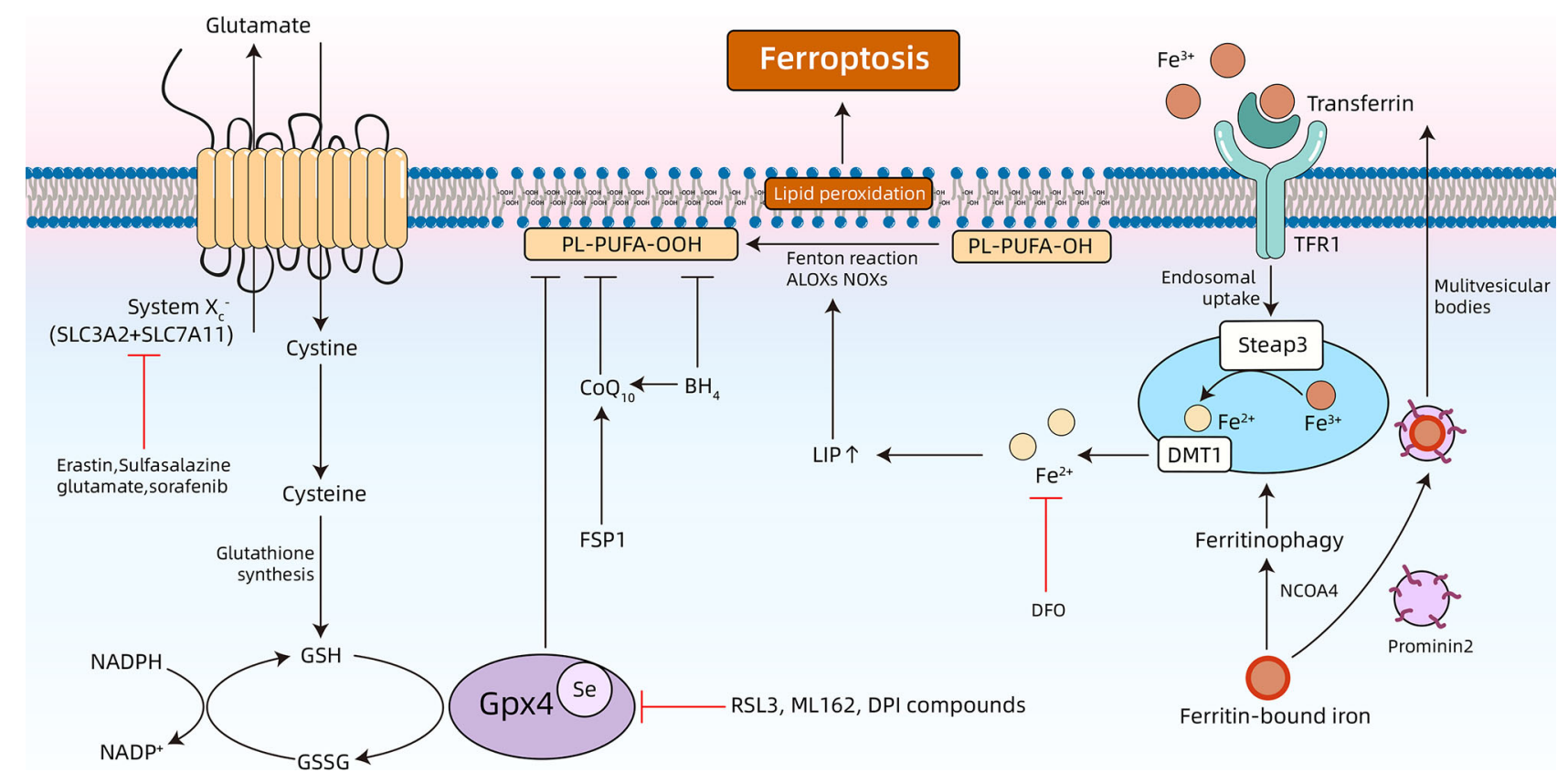

FIGURE 1 | Overview of the underlying mechanisms of ferroptosis. Ferroptosis is a type of programmed cell death that is characterized by accumulation of free iron and toxic lipid peroxides. Dysregulation of intracellular iron metabolism or glutathione peroxidation pathways leads to accumulation of lipid reactive oxygen species (ROS), and eventually cell death. Various inducers and inhibitors of ferroptosis are shown. TFR1, transferrin receptors 1; DFO, deferoxamine; NCOA4, nuclear receptor coactivator 4; ALOXs: arachidonate lipoxygenases; NADPH, nicotinamide adenine dinucleotide phosphate; NADP ${ }^{+}$, nicotinamide adenine dinucleotide phosphate; NOXs: Reduced form of NADPH oxidase; BH4, tetrahydrobiopterin; FSP1, ferroptosis suppressor protein 1; Se, selenocysteine; GSSG, oxidized GSH.

(DMT1), thereby increasing the concentration of the labile iron pool (LIP). Ferritinophagy refers to selective autophagy of ferritin and can modulate sensitivity to ferroptosis by adjusting LIP content (48-50). All the free iron of LIP facilitates ferroptosis by triggering formation of highly damaging hydroxyl radicals using the Fenton reaction, and catalyzing lipid peroxidation in the cellular membrane through lipoxygenase (LOXs) $(51,52)$. Under physiological conditions, the human body is able to maintain the homeostasis of intracellular iron. For example, intracellular iron can be effectively expelled to the outside of the cell using transferrin. Furthermore, prominin 2 can inhibit ferroptosis by promoting the formation of multivesicular bodies and exosomes (53).

\section{Lipid Metabolism}

Ferroptosis, a process that is heavily dependent on reactive oxygen species (ROS) and iron, is characterized by lipid peroxidation (54-56). Lipid peroxidation refers to the process in which oxidants (i.e., free radicals) abstract a labile hydrogen atom from the methylene group at the bisallyl position of polyunsaturated fatty acids (PUFAs), and PUFAs are substrates of pro-ferroptotic lipid peroxidation products including ACSL4 and LPCAT3 (54). Among the different classes of lipids in cells (i.e., fatty acids, phospholipids, cholesterols, cardiolipins, and sphingolipids), PUFAs containing bis-allylic protons vulnerable to hydrogen atom abstraction are the lipids that are most susceptible to oxidative damage in ferroptosis (57). After it was first noticed that ferroptosis is induced by RSL3 and erastin, further studies confirmed that this specially programmed cell death is driven by a loss of activity of the lipid repair enzyme glutathione (GSH)-glutathione peroxidase 4 (Gpx4) (Figure 1). Gpx4 is the sole selenoenzyme that is able to catalyze the conversion of toxic lipid hydroperoxides (L-OOH) into nontoxic lipid alcohols (L-OH) in a complex cellular membrane environment $(58,59)$. The deletion and inhibition of Gpx4 is able to facilitate lethal accumulation of lipid peroxides into the cellular membrane and initiate execution of ferroptosis. Therefore, Gpx4 is considered to be a central link in regulating ferroptosis (60). Furthermore, there are novel emerging ferroptosis-inducing compounds (i.e., ML162 and DPI compounds) that are able to induce lipid peroxidation by inhibiting Gpx4 function $(28,60)$. Moreover, lipophilic antioxidants, which include vitamin E, ferrostatin-1 (Fer-1), and liproxstatin-1 (Lip-1), are able to alleviate ferroptosis by inhibiting lipid autoxidation (61-63).

\section{Amino Acid and GSH Metabolism}

The core part of the amino acid-related mechanism of ferroptosis is the GSH-Gpx4 axis (Figure 1) (60). System $x_{c}^{-}$, a $\mathrm{Na}^{+}$independent cystine/glutamate antiporter, is a disulfide-linked heterodimer that consists of SLC3A2 and SLC7A11. System $x_{c}^{-}$ imports extracellular cystine into the cytoplasm and simultaneously exports intracellular glutamate into the extracellular compartment $(64,65)$. The intracellular cystine will be then converted to cysteine, which is further used to synthesize GSH (66). GSH, a tripeptide antioxidant, is then used 
as a cofactor for Gpx4, which reduces oxidative stress and maintains the intracellular redox balance. In addition to the canonical amino acid metabolism pathway that is mentioned above, coenzyme Q10 (CoQ10) works in parallel with Gpx4 in order to suppress phospholipid peroxidation and ferroptosis. Many molecules that induce ferroptosis by regulating amino acid metabolism have now been discovered. Erastin (28), sulfasalazine (SAS) (67), and sorafenib (68) are able to inhibit system $x_{c}^{-}$, which causes GSH depletion, thereby inducing ferroptotic cell death. $\beta$-mercaptoethanol is able to promote cystine uptake in order to inhibit erastin-stimulated ferroptosis in HT1080 cells (67). Acetaminophen-induced cell death is characterized by a depletion of GSH, and ferroptosis has been shown to be involved in this process (69). Importantly, the accumulation of extracellular glutamate is a natural trigger for inducing ferroptosis (60). Previous studies have indicated that excessive stimulation of glutamate is able to induce death of nerve cells ( 70 , 71). Glutamate-induced toxicity is an oxidative and irondependent process that is caused by calcium influx and competitive inhibition of system $x_{c}^{-}$, which ultimately induces ferroptosis by inhibiting Gpx4 $(60,70,71)$.

\section{EVIDENCE OF FERROPTOSIS IN CM}

Of note, previous studies summarized below have indicated that increased iron accumulation, lipid peroxidation in CM, and abnormal activity of amino acid, which suggests a relationship between CM and ferroptosis.

\section{Increased Iron Accumulation in the Brain With CM}

Iron is the most abundant trace metal present in organisms and is essential for both humans and pathogenic microbes $(72,73)$. Several central metabolic pathways, including oxygen transport, the tricarboxylic acid (TCA) cycle, and electron transport chains, cannot be done in the absence of iron (74). However, under pathological conditions, iron accumulation damages the body. Ferrous iron $\left(\mathrm{Fe}^{2+}\right)$ can be converted into ferric $\left(\mathrm{Fe}^{3+}\right)$ using Fenton reaction, and promotes ROS production and activates LOXs, simultaneously, which leads to oxidative damage $(75,76)$.

For CM, studies have indicated that there are elevated levels of ferritin in the CSF (23). Additional studies have suggested that ferritin is locally synthesized by brain cells, which reveals the involvement of ferritin is induced within the pathogenesis of CM in the brain. However, the specific mechanisms remain unclear (23). Furthermore, the increase of ferritin within the CSF is considered a measure to screen for meningitis (24). Compared to non-infectious neurologic disorders (i.e. seizure disorders and multiple sclerosis) and non-CM meningitis (i.e. viral and bacterial meningitis), the most significant increase of ferritin has been observed in CM (24). In this regard, compared to other types of meningitis, determining levels of CSF ferritin may be a diagnostic indicator, and CM may have a unique pathogenic mechanism involving iron metabolism. According to prior studies, the abundance of ferritin is a crucial factor that regulates sensitivity to ferroptosis $(48,50,55)$. Ferritin is able to release iron into LIP through ferritinophagy, which causes increased sensitivity to ferroptosis. Therefore, this peculiar irondependent cell death is likely triggered by high levels of ferritin.

Iron accumulation is able to aggravate the progression of CM. A study has suggested that iron overload exacerbates experimental CM (77). Within the CM model constructed by two strains of C. neoformans, Sb26 (a-Dserotype) and Sb26Rev (a-D serotype), FeDx treatment distinctly increased brain colony forming units (CFU). Additionally, median survival times (MSTs) were markedly decreased (77).

Jarvis and colleagues analyzed the local and systemic immune responses in patients with $\mathrm{HIV}$-associated CM and found that increased levels of proinflammatory cytokines and chemokines (i.e. IL-6, IFN- $\gamma$, and tumor necrosis factor (TNF)) are correlated with CSF macrophage activation, reduced fungal burden, rapid clearance of infection, and prognosis (78). However, these inflammatory cytokines are able to promote extracellular iron uptake by brain astrocytes and microglia by increasing the expression of the iron transporters divalent metal transporter 1 (DMT1) and Ferroportin 1 (FPN1) $(79,80)$. Moreover, current studies have validated that microglia have a vital function in controlling the growth of C. neoformans within the CNS (81). Consistent with prior work, IFN- $\gamma$ plus lipopolysaccharide (LPS) enhanced anticryptococcal activity by the murine microglial cell line BV-2 (82). Nevertheless, through in vitro treatment using ferric nitrilotriacetate (FeNTA), the effect of iron-loaded brain microglial cells on stimulation of IFN- $\gamma$ and LPS becomes significantly weakened (83). Therefore, excessive iron accumulation in the complex immune microenvironment of $\mathrm{CM}$ will affect effective cells' function, but the specific pathway is still unknown.

Therefore, based on these results, we can preliminarily conclude that iron metabolism within the brain can be disturbed in CM, which mainly manifests by increasing ferritin levels within CSF and accumulating iron in the brain. These disorders may further cause physiological immune dysfunction of the microglia. However, whether iron-dependent ferroptotic cell death is involved in the above process needs further elaboration.

\section{Lipid Peroxidation Is a Common Manifestation of CM and Ferroptosis}

Lipid peroxidation, a common hallmark of ferroptosis, is dependent on ROS $(54,56)$. ROS, which includes neutral molecules (i.e., hydrogen peroxide), ions (i.e., superoxide anion), or radicals (i.e., hydroxyl radicals), are mainly produced during cellular respiration and metabolic processes (84-86). ROS remain at homeostatic concentrations within mammalian cells and participates in specific normal physiological processes (86). In order to maintain the appropriate state of ROS, humans have various antioxidant defenses, which mainly include antioxidant enzymes (i.e. superoxide dismutase, catalase, and Gpx) and oxidation scavengers (i.e. vitamin E, ascorbic acid, carotenoids, and polyphenols) (86). However, ROS becomes excessively produced in certain diseases, and the antioxidants within the human body cannot keep them at their 
original homeostatic concentrations (54). ROS reacts readily with nucleic acids, proteins, and lipids at high concentrations, which eventually leads to DNA damage, protein denaturation, and lipid peroxidation (86). As oxidative stress is related to the processes of aging and cell death, ROS is involved in several age-related diseases (including $\mathrm{AD}, \mathrm{PD}$, and cardiovascular diseases), and these diseases are more or less confirmed to be related to ferroptosis (87-89). With regards to $\mathrm{CM}$, there is also evidence of lipid peroxidation taking place with in brain cells.

Several studies have identified that $C$. neoformans is able to cause lipid peroxidation in the vasculature and the brain (25). In 2010, a study showed that cellular lipid peroxidation levels of rabbits inoculated with $C$. neoformans increased significantly, and histopathological examination indicated that the oxidative stress of multinuclear phagocytes mainly manifests as large amounts of granular brown pigment within cells (25). The study also found that $C$. neoformans increases lipid peroxidation in the rabbit model, largely due to macrophagerelated oxidative stress and release of ROS (25). Not only that, but another animal experiment identified that thiobarbituric acid reactive substances (TBARS) in the brain are one of the most commonly utilized methods for measuring lipid peroxidation (26). The study discovered that, compared to the healthy group, lipid peroxidation levels in CM-infected rat brains were significantly increased (26). Analogously, when C. neoformans infects macrophages, significant levels of cellular lipid peroxidation occurs, and a higher volume density of dense lipid droplets within macrophages can be observed using an electron microscope (27). Studies that are related to HIV infection also provide evidence of lipid peroxidation and claim that lipid peroxidation may be linked to HIV-1-associated neurodegeneration $(90,91)$.

These results confirm the occurrence of lipid peroxidation in CM. However, overproduction of ROS and increased lipid peroxidation have severe impacts on cellular physiological functions, particularly within the CNS (92). Current studies have shown that high levels of ROS are able to react with PUFAs in various cellular membranes, which increases lipid peroxidation products, including 4-hydroxynonenal and malondialdehyde (93). These products have a long intracellular half-life and have been shown to have an effect on cellular activities that are related to neurite plasticity, which includes signal transduction pathways and ubiquitination (93). Additionally, an increasing number of neurological diseases have been shown to be related to increasing levels of lipid peroxidation and accumulation of lipid peroxidation products, such as neurodegenerative diseases (i.e., AD, PD, and amyotrophic lateral sclerosis) and CNS traumas (i.e. stroke, traumatic brain injury, and spinal cord injury) (92, 94, 95). Therefore, lipid peroxidation in CM can have an adverse effect on the CNS through the mechanisms mentioned prior.

In combination with the preceding descriptions of iron metabolism in CM, the mechanism of lipid peroxidation caused by cryptococcal infection of the brain is likely to, at least partly, include an iron-dependent process. However, this irondependent lipid peroxidation is a classic feature of ferroptosis.
Hence, ferroptosis is likely involved in the potential link between lipid peroxidation and iron accumulation in CM. However, more systematic studies that analyze the function of ferroptosis in the pathogenesis of CM are needed in order to extend and validate these initial observations.

\section{Affects the Amino Acid Metabolism of the Brain}

The effect of CM and C. neoformans on the host's amino acid metabolism has not yet been fully elucidated. Only a few studies have discovered that CM has an effect on glutamate and cysteine metabolism, which is closely related to ferroptosis. Metabolomics research has identified several potential metabolic biomarkers of CSF that can help distinguish different types of meningitis. According to different studies, glutamate and cysteine may be potential metabolic markers of CSF that can help distinguish CM from tuberculous meningitis (96). Similarly, another study evaluated the metabolic status of lung epithelial cells that were infected with C. neoformans. Analysis of these results indicates that several pathways, including glutamate metabolism and GSH metabolism, were impaired at the low multiplicity of infections (MOI) samples, and that incubation at higher MOI resulted in the perturbance of the cysteine metabolism (97). Importantly, extensive research has proven that these dysregulated amino acids have a significant role in ferroptosis induction. Glutamate is an important sensitizer of ferroptosis (60). As described above, a high extracellular concentration of glutamate inhibits system $x_{c}^{-}$ and induces ferroptosis, which likely explains the toxic effects of glutamate when it accumulates to higher concentrations within the CNS $(70,71,98)$. Indeed, animal experiments validated that glutamate, the neuroexcitatory amino acid, is involved in brain damage caused by cryptococcal infection (99). Researchers concluded that, as has been discovered with additional brain infectious diseases or toxic disorders, a depletion of glutamate in the brain of experimental murine cerebral cryptococcosis can be caused by excessive release of glutamate (99). However, accumulation of extracellular glutamate is thought to be a natural trigger for induction of ferroptosis (60).

Overall, although current research in amino acid metabolism of CM remains insufficient, combined with existing research and experimental results of iron metabolism and lipid metabolism, attention needs to be paid to the relationship between iron accumulation, lipid peroxidation, and dysregulated amino acid metabolism, all of which are hallmarks of ferroptosis. Hence, ferroptotic cell death is likely involved in the cellular damage of CM. Nevertheless, the current research remains inconclusive, and more in-depth research is needed.

\section{IMMUNOMODULATORY AND PRO- INFLAMMATORY ROLE OF FERROPTOSIS}

Since ferroptotic cells secrete DAMPs and alarmin, which are recognized by immune receptors and ultimately aggravate cell death and inflammation, ferroptosis is considered as both immunogenic and pro-inflammatory (100). In the model of 
folic acid-induced AKI, our study results concluded that ferroptosis can cause renal tubule damage by triggering inflammation, as upregulation of pro-inflammatory cytokines (interleukin-33, TNFa, MCP-1) and necroptotic proteins are quenched through Fer-1, a specific inhibitor of ferroptosis (101). Furthermore, inflammatory cells infiltrate into the ferroptotic tissue, which was noticeable, as determined by staining against F4/80, a $160 \mathrm{kD}$ glycoprotein expressed by murine macrophages (61). Furthermore, ferroptosis regulates synchronized tubular cell death and contributes to immunecell extravasation into the damaged tissue, while leukocyte transmigration and levels of pro-inflammatory cytokines are significantly decreased in the presence of Fer-1 (102). In an in vivo model of closed-chest myocardial ischemia-reperfusion injury (IRI), Fer-1 significantly reduced the initial infarct area and neutrophil infiltration into the heart, which suggests that immunogenicity and pro-inflammatory properties of ferroptosis are involved in early cardiomyocyte cell death and neutrophil recruitment in vivo (103). Results from this study also validated that ferroptotic cell death initiates neutrophil recruitment after heart transplantation through a TLR4/Trif-dependent pathway (103). These results reflect that ferroptosis can induce innate immunity and promote production of pro-inflammatory molecules in various diseases. However, the specific pathways through which ferroptosis carried out its immunogenic and proinflammatory effects have not yet been fully elucidated.

Arachidonic acid (AA) oxidation products, which are released from ferroptotic cells, are considered to be immunomodulatory signals and, therefore, regulate immunity. Eicosanoids are biologically active signaling lipids that are derived from AA and related PUFAs (104). Over the decades, inhibiting the formation or receptor-mediated actions of classical eicosanoids, such as through aspirin or other non-steroidal antiinflammatory drugs, remains the prevailing strategy to alleviate inflammation. Friedmann Angeli et al. demonstrated that inducible Gpx4 disruption triggers Necl-sensitive ferroptotic cell death, and determined that AA metabolites are released into the cell culture medium (61). Levels of eicosanoids, such as 5-hydroxyeicosatetraenoic acid (HETE), 11-HETE, and 15HETE, were increased upon triggering of ferroptosis (61). Concurrently, ferroptosis-inducing agents (FINs) also induced a similar HETE signature (61). Analogously, in heart ferroptotic cells, the abundance of several HETE, epoxyeicosatrienoic acid (EET) species, and prostaglandin D2 were significantly increased, and Fer-1 treatment resulted in a decrease of these lipid mediators (103). Additional studies have also validated that inhibition of ferroptosis led to a reduction in production of proinflammatory lipid mediators, and inhibited TNF- or IL-1mediated activation of the NF- $\mathrm{BB}$ pathway (105). These findings suggest that ferroptotic cells secrete specific lipid mediators that are involved in downstream signal transduction mechanisms.

Several studies have suggested that eicosanoids have an essential role in infection and inflammation and play a role in immunomodulation (106). For example, PGE2 leads to inhibition of necrotic cell death of macrophages, thus promoting pathogen resistance and host protection during $M$. tuberculosis infection (107). However, PGE2 impairs immunity to the influenza A virus by inhibiting macrophage antigen presentation and T-cell mediated immunity (108). Another example, despite the fact that definitive biological functions for HETE products have not yet been clarified, is that some may be ligands for peroxisome proliferator-activated receptor- $\alpha$ $(\operatorname{PPAR} \alpha)$ and PPAR $\gamma$, which induce anti-inflammatory effects $(109,110)$. Therefore, pro-inflammatory lipid mediators secreted by ferroptotic cells can further regulate immunity or drive secondary inflammatory damage in ferroptosisrelated pathologies.

In addition to lipid mediators, studies have also discovered that ferroptotic cells release high mobility group box 1 (HMGB1), which also mediates inflammation (111). HMGB1 is a nuclear protein that can be released by dead, dying, or injured cells (112). Once HMGB1 is released, it can bind to several receptors, including the toll-like receptor 4 (TLR4) and advanced glycosylation end-product specific receptor (AGER), in order to mediate immune responses (112). Thus, in order to measure the degree of HMGB1 release from ferroptotic cells, multiple cell lines, including HL-60 cells (a human leukemia cell line), HT1080 (a human fibrosarcoma cell line), PANC1 (a human pancreatic cancer cell line), and an immortalized MEF line, were treated with ferroptosis activators (erastin, sorafenib, RSL3, and FIN56) (111). Through ELISA analysis, it was discovered that HMGB1 released from these ferroptotic cells were significantly increased, and that this effect was hindered by ACSL4 knockdown and ferroptosis inhibitors, which include Fer-1, Lip-1, or baicalein (111). More importantly, this study demonstrated that ferroptotic cells are triggered by the proinflammatory cytokine TNF production in the bone marrowderived macrophages, while anti-HMGB1 neutralizing antibodies are able to attenuate TNF production, which suggests that HMGB1 mediates inflammation response in ferroptosis (111). Therefore, inhibiting release of HMGB1 from ferroptotic cells is a potential anti-inflammatory strategy that is utilized for ferroptosis-associated diseases.

HMGB1 is a critical element that is required for immunogenicity of cancer cells. The absence of HMGB1 expression in dying tumor cells compromises DC-dependent T-cell priming by tumor-associated antigens (113). Moreover, Wang et al. confirmed a direct link between ferroptosis and antitumor immunity (114). As mentioned above, the system xc- is a disulfide-linked heterodimer that consists of SLC3A2 and SLC7A11 and plays a key role in ferroptosis. IFN- $\gamma$ released from immunotherapy-activated CD8 $+\mathrm{T}$ cells downregulates SLC3A2 and SLC7A11 expression, and increases tumor cell lipid peroxidation and ferroptosis, which ultimately improves anti-tumor efficacy of immunotherapy (114). Therefore, regulating ferroptosis-associated metabolism in tumors is a potential strategy to improve the efficacy of cancer immunotherapy in the future.

For CM, confocal microscopy results indicated that $C$. neoformans induces translocation of HMGB1 in brain endothelial cells, which, in turn, results in endothelial cell 
damage and ultimately promotes breakdown of the blood-brain barrier (BBB) (115). Given the role of HMGB1 in inflammation and immune regulation, regulating ferroptosis may be an important method to suppress the outbreak of inflammation in particular infectious diseases, including CM.

\section{FERROPTOSIS AND INFECTIOUS DISEASES}

As mentioned prior, ferroptosis is a type of programmed cell death that is characterized by free iron accumulation and toxic lipid peroxides. In infectious diseases, cell death is the most common phenomenon. Furthermore, it has been validated that ferroptotic cell death may be the main form of host cell death caused by specific pathogens. Additionally, ferroptosis of immune cells during infection is advantageous for infectious agents. Amaral et al. identified that M. tuberculosis-induced macrophage death in vitro is a type of necrosis, as opposed to apoptosis or pyroptosis, and that this form of cell death is accompanied by increased levels of intracellular labile iron and membrane lipid peroxides, as well as a decrease in GSH and Gpx4 expression levels (39). Interestingly, all of the significant parameters that were covered above are key hallmarks of ferroptotic cell death. Therefore, studies have investigated whether M. tuberculosis-induced death of macrophages is related to ferroptosis. In fact, results from these studies have confirmed that ferroptosis inhibitors, including pyridoxal isonicotinoyl hydrazone (PIH) and Fer-1, suppress lipid peroxidation and cell death, suggesting that ferroptosis may be involved in this form of necrosis (39). Importantly, these results were further confirmed by employing a mice model of $M$. tuberculosis infection (39). P. aeruginosa, a common gramnegative rod-shaped bacterium, is able to synthesize LOXs, which converts arachidonic acid in the membrane of the host cell to 15-hydroxyeicosatetraenoic acid, leading to the induction of lipid peroxidation and acting as an executor in ferroptosis $(35,36)$.

Similarly, another study discovered that macrophages infected with Histoplasma capsulatum, an environmentallyacquired fungal pathogen, can also lead to increased intracellular oxidation products, and eventually cell death (116). Despite the fact that the ferroptosis inhibitor Fer-1 can reduce the cell death caused by $H$. capsulatum infection, further experiments have validated that the mechanism decreases fungal ergosterol synthesis, instead of inhibiting ferroptosis (116). Consequently, upon exploring the relationship between ferroptotic cell death and pathogenic mechanism of pathogens, attention needs to be paid to ferroptosis inhibitors, especially Fer-1, which may have a direct inhibitory effect on pathogens. Furthermore, fungal infection can also be caused by plant ferroptotic cell death (117-119). Ceratocystis Fimbriata BMPZ13 infection of sweet potato leads to iron-associated ferroptotic cell death in leaves and veins (119). Incompatible rice (Oryza sativa)-Magnaporthe oryzae interactions induces ferroptotic cell death in rice cells (118).
At the end of 2019, coronavirus disease-2019 (COVID-19), caused by SARS CoV-2, started to spread worldwide. Several studies have further elaborated that cellular redox imbalance and iron incoordination can play an essential role in the pathogenesis of COVID-19 (41, 42). As an example, COVID-19 infection induces secretion of IL-6, which can stimulate ferritin and synthesis of hepcidin, eventually leading to iron dysregulation (40). Excess intracellular iron interacts with molecular oxygen in order to generate ROS through the Fenton reaction, which results in mitochondrial function disorder and ferroptotic cell death (40). A recent study also suggested that the mechanism of SARS CoV-2, leading to ferroptotic cell death, may be related to cysteine-associated cellular metabolism (42). Although the relative research appears sparse, these results suggest new potential links between ferroptosis and COVID-19, which may represent a novel strategy for treatment of COVID-19.

Moreover, with regards to $\mathrm{HBV}$ infection, liver fibrosis and hepatocellular carcinoma (HCC) are the most severe stages of disease progression (120). Hepatitis B virus X protein $(\mathrm{HBx})$ plays a significant role in $\mathrm{HBV}$ replication and the development of HCC (43). Furthermore, HBx can further activate hepatic stellate cells (HSCs) by inhibiting ER stress, ferroptosis, and ultimately promoting liver fibrosis. In contrast, chrysophanol, which is derived from a Chinese herb, reverses this inhibition and may be a possible therapeutic strategy for treatment of antihepatic fibrosis (121). However, the relationship between HBxinduced ferroptosis and liver fibrosis needs to be further investigated.

In conclusion, these studies have indicated signs of ferroptosis across many infectious diseases; however, specific links need to be further researched. Existing evidence has validated that ferroptosis of immune cells during infection is beneficial to disease progression, and that ferroptotic cell death can induce release of DAMPs, which can both aggravate cell death and inflammation. Therefore, in order to control inflammation and cell death during infection, an effective regulation of ferroptosis may be a novel and possible strategy for treatment of infectious diseases.

\section{DISCUSSION AND FUTURE PERSPECTIVES}

Since the majority of infectious diseases involve well-characterized cellular necrosis and pro-inflammatory mechanisms, these represent possible new targets for therapeutic intervention. In this review, we elaborate on possible potential links between CM and ferroptosis. As iron overload and lipid peroxidation are fundamental characteristics of ferroptosis, these hallmarks are further reflected in CM. Hence, we reason that there might be a relationship between this cell death modality and CM.

Ferroptosis, a non-apoptotic form of cell death, is characterized by iron-dependent lipid peroxidation. For CM, an increase of ferritin within the CSF and accumulation of iron in the brain cells may indicate induction of ferroptosis. Ferritin's abundance is a crucial factor that governs ferroptosis sensitivity 
as ferritin can release iron into the LIP through ferritinophagy, thereby causing increased sensitivity to ferroptosis (48-50). Importantly, intracellular iron accumulation and lipid peroxidation are able to coexist, and ferroptosis can exactly connect them together (Figure 2). Therefore, according to all current research results, we hypothesize that ferroptosis is likely involved in the pathogenic mechanism of $\mathrm{CM}$, similar to other infectious diseases. However, we still need more in-depth research in order to validate this hypothesis. Through regulation of ferroptosis, we are able to better understand the relationship between iron accumulation and lipid peroxidation and ferroptosis. However, we need to pay more attention to the direct inhibitory effect of the regulator on pathogens. One study pointed out that Fer-1, a commonly used ferroptosis-inhibiting agent, reduced growth of $C$. neoformans in the YNB media by $>95 \%$ (116). Hence, when designing an experiment, we should be able to regulate ferroptosis through multiple pathways.

In addition to ferroptosis, regulated necrosis is also related to pyroptosis and necroptosis. Recently, studies have identified that ferroptosis and pyroptosis may intersect (122). Therefore, in cellular necrosis in C. neoformans infection, it is necessary to exclude intersections with additional cell death pathways. Furthermore, ferroptosis is immunogenic and proinflammatory as ferroptotic cells release DAMPs and alarmin, which may be recognized by immune receptors and ultimately aggravate cell death and inflammation (100). Despite the fact that no studies have explored the expression of ferroptosisassociated DAMPs in CM, due to the extensive role of ferroptosis in the pro-inflammatory and immune regulation, this will also become a direction for future designed experiments. Overall, more systematic studies that analyze the function of ferroptosis in the pathogenesis of CM are needed in order to extend and validate these initial observations.

Moreover, iron accumulation may be an indicator of differential diagnosis of $\mathrm{CM}$ and helps estimate the severity of disease. Compared to non-infectious neurologic disorders and meningitis, other than $\mathrm{CM}$, the increase in ferritin is most significant in CM, and iron overload can help exacerbate experimental $\mathrm{CM}$ and significantly weaken the function of microglia. Beyond that, overproduction of ROS and increased

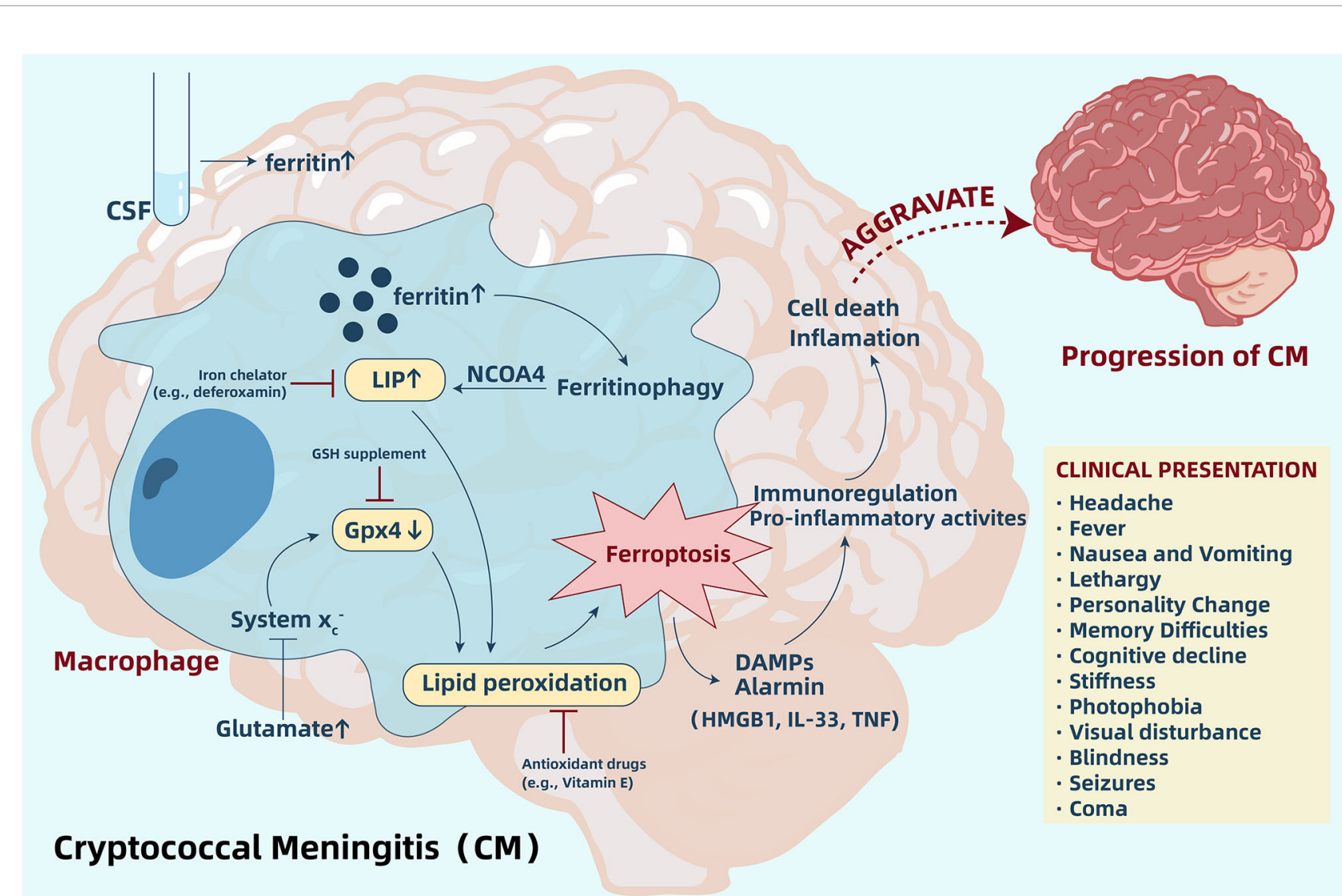

FIGURE 2 | Ferroptosis aggravates cryptococcal meningitis (CM) by regulating immunity and pro-inflammatory activity. In CM, increased ferritin levels within the CSF and accumulation of iron in brain cells release iron into LIP through ferritinophagy, and increased glutamate may inhibit System xc-, which leads to a depletion of Gpx4 in cells. Accumulation of iron in LIP and depletion of Gpx4 induces lipid peroxidation and further triggers ferroptosis. Ferroptotic cells release DAMPs and alarmin, which participate in immune regulation and pro-inflammatory activities, ultimately aggravating cell death and inflammation. Regulating ferroptosis by inhibitors, such as iron chelators and antioxidants, may be a potential novel strategy to suppress the pathway and delay CM progression. CSF, cerebrospinal fluid; LIP, labile iron pool; GSH, glutathione; Gpx4, glutathione peroxidase 4; DAMPs, damage-associated molecular pattern molecules; HMGB1, high mobility group box 1; IL-33, interleukin-33; TNF, tumor necrosis factor. 
lipid peroxidation also severely impacts cellular physiological functions, particularly within the CNS cells. Therefore, the mechanism of iron metabolism and lipid metabolism in CM may be a potential new direction for future research.

Ferroptosis has been considered a novel target for therapeutic intervention. An increasing number of studies have established that ferroptotic cell death is closely related to numerous diseases, particularly in tumors. In recent years, the role of ferroptosis in infectious diseases has gradually emerged. As mentioned prior, M. tuberculosis induced iron accumulation and lipid peroxidation of macrophages and confirmed ferroptotic cell death, which can be suppressed by the ferroptosis regulator Fer-1 and iron chelation (39). P. aeruginosa utilizes host polyunsaturated phosphatidylethanolamines in order to induce theft-ferroptosis within the bronchial epithelium and may represent a possible therapeutic target against $P$. aeruginosarelated diseases (35). SARS CoV-2 may also trigger ferroptosis by promoting release of IL- 6 and affecting cysteine metabolism, which suggests a novel strategy for COVID-19 treatment (4042). This evidence indicates that ferroptosis is involved in pathogenesis of various pathogens, and inhibition of this unique form of cell death may be a novel treatment strategy. Unfortunately, there is no direct or indirect link between ferroptosis regulators and C. neoformans and CM. However, additional studies have been required to validate ferroptosis as a viable strategy for $\mathrm{CM}$ treatment. Nevertheless, prior to this, more research is needed to elucidate the status of ferroptosis in

\section{REFERENCES}

1. Park BJ, Wannemuehler KA, Marston BJ, Govender N, Pappas PG, Chiller TM. Estimation of the current global burden of cryptococcal meningitis among persons living with HIV/AIDS. AIDS (London England) (2009) 23 (4):525-30. doi: 10.1097/QAD.0b013e328322ffac

2. Rajasingham R, Smith RM, Park BJ, Jarvis JN, Govender NP, Chiller TM, et al. Global burden of disease of HIV-associated cryptococcal meningitis: an updated analysis. The Lancet. Infect Dis (2017) 17(8):873-81. doi: 10.1016/ S1473-3099(17)30243-8

3. Tenforde MW, Gertz AM, Lawrence DS, Wills NK, Guthrie BL, Farquhar C, et al. Mortality from HIV-associated meningitis in sub-Saharan Africa: a systematic review and meta-analysis. J Int AIDS Soc (2020) 23(1):e25416. doi: 10.1002/jia2.25416

4. Yuchong C, Fubin C, Jianghan C, Fenglian W, Nan X, Minghui Y, et al. Cryptococcosis in China (1985-2010): review of cases from Chinese database. Mycopathologia (2012) 173(5-6):329-35. doi: 10.1007/s11046-011-9471-1

5. Marr KA, Datta K, Pirofski L-A, Barnes R. Cryptococcus gattii infection in healthy hosts: a sentinel for subclinical immunodeficiency? Clin Infect Dis (2012) 54(1):153-4. doi: 10.1093/cid/cir756

6. Maziarz EK, Perfect JR. Cryptococcosis. Infect Dis Clinics North Am (2016) 30(1):179-206. doi: 10.1016/j.idc.2015.10.006

7. Sirinavin S, Intusoma U, Tuntirungsee S. Mother-to-child transmission of cryptococcus neoformans. Pediatr Infect Dis J (2004) 23(3):278-9. doi: 10.1097/01.inf.0000115639.43305.9b

8. Spec A, Powderly WG. Cryptococcal meningitis in AIDS. Handb Clin Neurol (2018) 152:139-50. doi: 10.1016/B978-0-444-63849-6.00011-6

9. Zaragoza O. Basic principles of the virulence of. Virulence (2019) 10(1):490501. doi: 10.1080/21505594.2019.1614383

10. Casadevall A, Rosas AL, Nosanchuk JD. Melanin and virulence in Cryptococcus neoformans. Curr Opin Microbiol (2000) 3(4):354-8. doi: 10.1016/S1369-5274(00)00103-X the pathological mechanisms of CM. In this review, we provide a promising research direction, and the potential to decrease tissue damage while reducing pathogenic burden is an attractive aspect of this research direction.

\section{DATA AVAILABILITY STATEMENT}

The original contributions presented in the study are included in the article/supplementary material. Further inquiries can be directed to the corresponding authors.

\section{AUTHOR CONTRIBUTIONS}

All the authors participated in analyzing and discussing the literature, commenting on, and approving the manuscript. AS and JS supervised the research, led the discussion, and wrote and revised the manuscript. All authors contributed to the article and approved the submitted version.

\section{FUNDING}

This work was funded by the National Natural Science Foundation of China (81670567 and 81701144).
11. Kwon-Chung KJ, Rhodes JC. Encapsulation and melanin formation as indicators of virulence in Cryptococcus neoformans. Infect Immun (1986) 51(1):218-23. doi: 10.1128/IAI.51.1.218-223.1986

12. Wang Y, Aisen P, Casadevall A. Cryptococcus neoformans melanin and virulence: mechanism of action. Infect Immun (1995) 63(8):3131-6. doi: 10.1128/IAI.63.8.3131-3136.1995

13. Polacheck I, Hearing VJ, Kwon-Chung KJ. Biochemical studies of phenoloxidase and utilization of catecholamines in Cryptococcus neoformans. J Bacteriol (1982) 150(3):1212-20. doi: 10.1128/ JB.150.3.1212-1220.1982

14. Nosanchuk JD, Casadevall A. Cellular charge of Cryptococcus neoformans: contributions from the capsular polysaccharide, melanin, and monoclonal antibody binding. Infect Immun (1997) 65(5):1836-41. doi: 10.1128/ IAI.65.5.1836-1841.1997

15. Franco-Paredes C, Womack T, Bohlmeyer T, Sellers B, Hays A, Patel K, et al. Management of Cryptococcus gattii meningoencephalitis. Lancet Infect Dis (2015) 15(3):348-55. doi: 10.1016/S1473-3099(14)70945-4

16. Perfect JR, Dismukes WE, Dromer F, Goldman DL, Graybill JR, Hamill RJ, et al. Clinical practice guidelines for the management of cryptococcal disease: 2010 update by the infectious diseases society of america. Clin Infect Dis (2010) 50(3):291-322. doi: 10.1086/649858

17. Gowri M, Jayashree B, Jeyakanthan J, Girija EK. Sertraline as a promising antifungal agent: inhibition of growth and biofilm of Candida auris with special focus on the mechanism of action in vitro. J Appl Microbiol (2020) 128(2):426-37. doi: 10.1111/jam.14490

18. Treviño-Rangel RDJ, Villanueva-Lozano $\mathrm{H}$, Hernández-Rodríguez $\mathrm{P}$, Martínez-Reséndez MF, García-Juárez J, Rodríguez-Rocha $\mathrm{H}$, et al. Activity of sertraline against Cryptococcus neoformans: in vitro and in vivo assays. Med Mycol (2016) 54(3):280-6. doi: 10.1093/mmy/ myv109

19. Treviño-Rangel RDJ, Villanueva-Lozano H, Méndez-Galomo KS, SolísVillegas EM, Becerril-García MA, Montoya AM, et al. In vivo evaluation 
of the antifungal activity of sertraline against Aspergillus fumigatus. J Antimicrob Chemother (2019) 74(3):663-6. doi: 10.1093/jac/dky455

20. Katende A, Mbwanji G, Faini D, Nyuri A, Kalinjuma AV, Mnzava D, et al. Short-course amphotericin B in addition to sertraline and fluconazole for treatment of HIV-associated cryptococcal meningitis in rural Tanzania. Mycoses (2019) 62(12):1127-32. doi: 10.1111/myc.12995

21. Rhein J, Huppler Hullsiek K, Tugume L, Nuwagira E, Mpoza E, Evans EE, et al. Adjunctive sertraline for HIV-associated cryptococcal meningitis: a randomised, placebo-controlled, double-blind phase 3 trial. Lancet Infect Dis (2019) 19(8):843-51. doi: 10.1016/S1473-3099(19)30127-6

22. Villanueva-Lozano H, Treviño-Rangel RDJ, González GM, HernándezRodríguez PA, Camacho-Ortiz A, Castillo-Reyna L, et al. Clinical evaluation of the antifungal effect of sertraline in the treatment of cryptococcal meningitis in HIV patients: a single Mexican center experience. Infection (2018) 46(1):25-30. doi: 10.1007/s15010-017-1059-3

23. Deisenhammer F, Miller RF, Brink NS, Harrison MJ, Thompson EJ. 005Cerebrospinal fluid ferritin in HIV infected patients with acute neurological episodes. Genitourinary Med (1997) 73(3):181-3. doi: 10.1136/sti.73.3.181

24. Campbell DR, Skikne BS, Cook JD. 007Cerebrospinal fluid ferritin levels in screening for meningism. Arch Neurol (1986) 43(12):1257-60. doi: 10.1001/ archneur.1986.00520120041014

25. Hall CJ, Bouhafs L, Dizcfalusy U, Sandstedt K. Cryptococcus neoformans causes lipid peroxidation; therefore it is a potential inducer of atherogenesis. Mycologia (2010) 102(3):546-51. doi: 10.3852/08-110

26. De Azevedo MI, Ferreiro L, Da Silva AS, Tonin AA, Thorstenberg ML, Catilhos LG, et al. Cholinesterase of rats experimentally infected by Cryptococcus neoformans: Relationship between inflammatory response and pathological findings. Pathol Res Pract (2015) 211(11):851-7. doi: 10.1016/j.prp.2015.08.005

27. Gross NT, Hultenby K, Mengarelli S, Camner P, Jarstrand C. Lipid peroxidation by alveolar macrophages challenged with Cryptococcus neoformans, Candida albicans or Aspergillus fumigatus. Med Mycol (2000) 38(6):443-9. doi: 10.1080/714030972

28. Dixon SJ, Lemberg KM, Lamprecht MR, Skouta R, Zaitsev EM, Gleason CE, et al. Ferroptosis: an iron-dependent form of nonapoptotic cell death. Cell (2012) 149(5):1060-72. doi: 10.1016/j.cell.2012.03.042

29. Park E-J, Park Y-J, Lee SJ, Lee K, Yoon C. Whole cigarette smoke condensates induce ferroptosis in human bronchial epithelial cells. Toxicol Lett (2019) 303:55-66. doi: 10.1016/j.toxlet.2018.12.007

30. Yoshida M, Minagawa S, Araya J, Sakamoto T, Hara H, Tsubouchi K, et al. Involvement of cigarette smoke-induced epithelial cell ferroptosis in COPD pathogenesis. Nat Commun (2019) 10(1):3145. doi: 10.1038/s41467-01910991-7

31. Speer RE, Karuppagounder SS, Basso M, Sleiman SF, Kumar A, Brand D, et al. Hypoxia-inducible factor prolyl hydroxylases as targets for neuroprotection by "antioxidant" metal chelators: From ferroptosis to stroke. Free Radic Biol Med (2013) 62:26-36. doi: 10.1016/ j.freeradbiomed.2013.01.026

32. Weiland A, Wang Y, Wu W, Lan X, Han X, Li Q, et al. Ferroptosis and Its Role in Diverse Brain Diseases. Mol Neurobiol (2019) 56(7):4880-93. doi: 10.1007/s12035-018-1403-3

33. Do Van B, Gouel F, Jonneaux A, Timmerman K, Gelé P, Pétrault M, et al. Ferroptosis, a newly characterized form of cell death in Parkinson's disease that is regulated by PKC. Neurobiol Dis (2016) 94:169-78. doi: 10.1016/ j.nbd.2016.05.011

34. Hambright WS, Fonseca RS, Chen L, Na R, Ran Q. Ablation of ferroptosis regulator glutathione peroxidase 4 in forebrain neurons promotes cognitive impairment and neurodegeneration. Redox Biol (2017) 12:8-17. doi: 10.1016/j.redox.2017.01.021

35. Dar HH, Tyurina YY, Mikulska-Ruminska K, Shrivastava I, Ting H-C, Tyurin VA, et al. Pseudomonas aeruginosa utilizes host polyunsaturated phosphatidylethanolamines to trigger theft-ferroptosis in bronchial epithelium. J Clin Invest (2018) 128(10):4639-53. doi: 10.1172/JCI99490

36. Vance RE, Hong S, Gronert K, Serhan CN, Mekalanos JJ. The opportunistic pathogen Pseudomonas aeruginosa carries a secretable arachidonate 15lipoxygenase. Proc Natl Acad Sci U S A (2004) 101(7):2135-9. doi: 10.1073/ pnas.0307308101
37. Andrade BB, Pavan Kumar N, Mayer-Barber KD, Barber DL, Sridhar R, Rekha VVB, et al. Plasma heme oxygenase-1 levels distinguish latent or successfully treated human tuberculosis from active disease. PLoS One (2013) 8(5):e62618. doi: 10.1371/journal.pone.0062618

38. Costa DL, Namasivayam S, Amaral EP, Arora K, Chao A, Mittereder LR, et al. Pharmacological Inhibition of Host Heme Oxygenase-1 Suppresses Mycobacterium tuberculosis Infection In Vivo by a Mechanism Dependent on T Lymphocytes. mBio (2016) 7(5):e01675-16. doi: 10.1128/mBio.01675-16

39. Amaral EP, Costa DL, Namasivayam S, Riteau N, Kamenyeva O, Mittereder $\mathrm{L}$, et al. A major role for ferroptosis in Mycobacterium tuberculosis-induced cell death and tissue necrosis. J Exp Med (2019) 216(3):556-70. doi: 10.1084/ jem.20181776

40. Edeas M, Saleh J, Peyssonnaux C. Iron: Innocent bystander or vicious culprit in COVID-19 pathogenesis? Int J Infect Dis (2020) 97:303-5. doi: 10.1016/ j.ijid.2020.05.110

41. Muhoberac BB. What Can Cellular Redox, Iron, and Reactive Oxygen Species Suggest About the Mechanisms and Potential Therapy of COVID19? Front Cell Infect Microbiol (2020) 10:569709. doi: 10.3389/ fcimb.2020.569709

42. Singh Y, Gupta G, Kazmi I, Al-Abbasi FA, Negi P, Chellappan DK, et al. SARS CoV-2 aggravates cellular metabolism mediated complications in COVID-19 infection. Dermatol Ther (2020) 33(6):e13871. doi: 10.1111/ dth. 13871

43. Zhang Y, Liu H, Yi R, Yan T, He Y, Zhao Y, et al. Hepatitis B virus whole-X and X protein play distinct roles in HBV-related hepatocellular carcinoma progression. J Exp Clin Cancer Res (2016) 35(1):87. doi: 10.1186/s13046-0160366-3

44. Tang B, Zhu J, Li J, Fan K, Gao Y, Cheng S, et al. The ferroptosis and ironmetabolism signature robustly predicts clinical diagnosis, prognosis and immune microenvironment for hepatocellular carcinoma. Cell Commun Signal (2020) 18(1):174. doi: 10.1186/s12964-020-00663-1

45. Dolma S, Lessnick SL, Hahn WC, Stockwell BR. Identification of genotypeselective antitumor agents using synthetic lethal chemical screening in engineered human tumor cells. Cancer Cell (2003) 3(3):285-96. doi: 10.1016/S1535-6108(03)00050-3

46. Yang WS, Stockwell BR. Synthetic lethal screening identifies compounds activating iron-dependent, nonapoptotic cell death in oncogenic-RASharboring cancer cells. Chem Biol (2008) 15(3):234-45. doi: 10.1016/ j.chembiol.2008.02.010

47. Gao M, Monian P, Quadri N, Ramasamy R, Jiang X. Glutaminolysis and Transferrin Regulate Ferroptosis. Mol Cell (2015) 59(2):298-308. doi: 10.1016/j.molcel.2015.06.011

48. Mancias JD, Wang X, Gygi SP, Harper JW, Kimmelman AC. Quantitative proteomics identifies NCOA4 as the cargo receptor mediating ferritinophagy. Nature (2014) 509(7498):105-9. doi: 10.1038/nature13148

49. Gao M, Monian P, Pan Q, Zhang W, Xiang J, Jiang X. Ferroptosis is an autophagic cell death process. Cell Res (2016) 26(9):1021-32. doi: 10.1038/ cr.2016.95

50. Hou W, Xie Y, Song X, Sun X, Lotze MT, Zeh HJ, et al. Autophagy promotes ferroptosis by degradation of ferritin. Autophagy (2016) 12(8):1425-8. doi: 10.1080/15548627.2016.1187366

51. Shah R, Shchepinov MS, Pratt DA. Resolving the Role of Lipoxygenases in the Initiation and Execution of Ferroptosis. ACS Cent Sci (2018) 4(3):38796. doi: 10.1021/acscentsci.7b00589

52. Wenzel SE, Tyurina YY, Zhao J, St Croix CM, Dar HH, Mao G, et al. PEBP1 Wardens Ferroptosis by Enabling Lipoxygenase Generation of Lipid Death Signals. Cell (2017) 171(3):628-41.e26. doi: 10.1016/j.cell.2017.09.044

53. Brown CW, Amante JJ, Chhoy P, Elaimy AL, Liu H, Zhu LJ, et al. Prominin2 Drives Ferroptosis Resistance by Stimulating Iron Export. Dev Cell (2019) 51 (5):575-86.e4. doi: 10.1016/j.devcel.2019.10.007

54. Kajarabille N, Latunde-Dada GO. Programmed Cell-Death by Ferroptosis: Antioxidants as Mitigators. Int J Mol Sci (2019) 20(19):4968. doi: 10.3390/ ijms20194968

55. Latunde-Dada GO. Ferroptosis: Role of lipid peroxidation, iron and ferritinophagy. Biochimica et biophysica acta. Gen Subj (2017) 1861 (8):1893-900. doi: 10.1016/j.bbagen.2017.05.019

56. Yang WS, Stockwell BR. Ferroptosis: Death by Lipid Peroxidation. Trends Cell Biol (2016) 26(3):165-76. doi: 10.1016/j.tcb.2015.10.014 
57. Yang WS, Kim KJ, Gaschler MM, Patel M, Shchepinov MS, Stockwell BR. Peroxidation of polyunsaturated fatty acids by lipoxygenases drives ferroptosis. Proc Natl Acad Sci U S A (2016) 113(34):E4966-75. doi: 10.1073/pnas.1603244113

58. Yant LJ, Ran Q, Rao L, Van Remmen H, Shibatani T, Belter JG, et al. The selenoprotein GPX4 is essential for mouse development and protects from radiation and oxidative damage insults. Free Radical Biol Med (2003) 34 (4):496-502. doi: 10.1016/\$0891-5849(02)01360-6

59. Seiler A, Schneider M, Förster H, Roth S, Wirth EK, Culmsee C, et al. Glutathione peroxidase 4 senses and translates oxidative stress into 12/15lipoxygenase dependent- and AIF-mediated cell death. Cell Metab (2008) 8 (3):237-48. doi: 10.1016/j.cmet.2008.07.005

60. Yang WS, Sriramaratnam R, Welsch ME, Shimada K, Skouta R, Viswanathan VS, et al. Regulation of ferroptotic cancer cell death by GPX4. Cell (2014) 156(1-2):317-31. doi: 10.1016/j.cell.2013.12.010

61. Friedmann Angeli JP, Schneider M, Proneth B, Tyurina YY, Tyurin VA, Hammond VJ, et al. Inactivation of the ferroptosis regulator Gpx4 triggers acute renal failure in mice. Nat Cell Biol (2014) 16(12):1180-91. doi: $10.1038 /$ ncb3064

62. Shah R, Margison K, Pratt DA. The Potency of Diarylamine RadicalTrapping Antioxidants as Inhibitors of Ferroptosis Underscores the Role of Autoxidation in the Mechanism of Cell Death. ACS Chem Biol (2017) 12 (10):2538-45. doi: 10.1021/acschembio.7b00730

63. Feng H, Stockwell BR. Unsolved mysteries: How does lipid peroxidation cause ferroptosis? PLoS Biol (2018) 16(5):e2006203. doi: 10.1371/ journal.pbio. 2006203

64. Bridges RJ, Natale NR, Patel SA. System $\mathrm{xc}^{-}$cystine/glutamate antiporter: an update on molecular pharmacology and roles within the CNS. $\mathrm{Br} J$ Pharmacol (2012) 165(1):20-34. doi: 10.1111/j.1476-5381.2011.01480.x

65. Sato H, Tamba M, Ishii T, Bannai S. Cloning and expression of a plasma membrane cystine/glutamate exchange transporter composed of two distinct proteins. J Biol Chem (1999) 274(17):11455-8. doi: 10.1074/jbc.274.17.11455

66. Stockwell BR, Friedmann Angeli JP, Bayir H, Bush AI, Conrad M, Dixon SJ, et al. Ferroptosis: A Regulated Cell Death Nexus Linking Metabolism, Redox Biology, and Disease. Cell (2017) 171(2):273-85. doi: 10.1016/ j.cell.2017.09.021

67. Gout PW, Buckley AR, Simms CR, Bruchovsky N. Sulfasalazine, a potent suppressor of lymphoma growth by inhibition of the $\mathrm{x}(\mathrm{c})$ - cystine transporter: a new action for an old drug. Leukemia (2001) 15(10):163340. doi: $10.1038 /$ sj.leu.2402238

68. Dixon SJ, Patel DN, Welsch M, Skouta R, Lee ED, Hayano M, et al. Pharmacological inhibition of cystine-glutamate exchange induces endoplasmic reticulum stress and ferroptosis. eLife (2014) 3:e02523. doi: 10.7554/eLife. 02523

69. Lörincz T, Jemnitz K, Kardon T, Mandl J, Szarka A. Ferroptosis is Involved in Acetaminophen Induced Cell Death. Pathol Oncol Res: POR (2015) 21 (4):1115-21. doi: 10.1007/s12253-015-9946-3

70. Choi DW. Glutamate neurotoxicity and diseases of the nervous system. Neuron (1988) 1(8):623-34. doi: 10.1016/0896-6273(88)90162-6

71. Mehta A, Prabhakar M, Kumar P, Deshmukh R, Sharma PL. Excitotoxicity: bridge to various triggers in neurodegenerative disorders. Eur J Pharmacol (2013) 698(1-3):6-18. doi: 10.1016/j.ejphar.2012.10.032

72. Becker KW, Skaar EP. Metal limitation and toxicity at the interface between host and pathogen. FEMS Microbiol Rev (2014) 38(6):1235-49. doi: 10.1111/ 1574-6976.12087

73. Gerwien F, Skrahina V, Kasper L, Hube B, Brunke S. Metals in fungal virulence. FEMS Microbiol Rev (2018) 42(1):fux050. doi: 10.1093/femsre/ fux 050

74. Andreini C, Bertini I, Cavallaro G, Holliday GL, Thornton JM. Metal ions in biological catalysis: from enzyme databases to general principles. $J$ Biol Inorganic Chem: JBIC: Publ Soc Biol Inorganic Chem (2008) 13(8):1205-18. doi: 10.1007/s00775-008-0404-5

75. Papanikolaou G, Pantopoulos K. Iron metabolism and toxicity. Toxicol Appl Pharmacol (2005) 202(2):199-211. doi: 10.1016/j.taap.2004.06.021

76. Winterbourn CC. Toxicity of iron and hydrogen peroxide: the Fenton reaction. Toxicol Lett (1995) 82-83:969-74. doi: 10.1016/0378-4274(95) 03532-x
77. Barluzzi R, Saleppico S, Nocentini A, Boelaert JR, Neglia R, Bistoni F, et al. Iron overload exacerbates experimental meningoencephalitis by Cryptococcus neoformans. J Neuroimmunol (2002) 132(1-2):140-6. doi: 10.1016/S01655728(02)00324-7

78. Jarvis JN, Meintjes G, Bicanic T, Buffa V, Hogan L, Mo S, et al. Cerebrospinal fluid cytokine profiles predict risk of early mortality and immune reconstitution inflammatory syndrome in HIV-associated cryptococcal meningitis. PLoS Pathog (2015) 11(4):e1004754. doi: 10.1371/ journal.ppat.1004754

79. Urrutia P, Aguirre P, Esparza A, Tapia V, Mena NP, Arredondo M, et al. 009Inflammation alters the expression of DMT1, FPN1 and hepcidin, and it causes iron accumulation in central nervous system cells. J Neurochem (2013) 126(4):541-9. doi: 10.1111/jnc.12244

80. Mccarthy RC, Sosa JC, Gardeck AM, Baez AS, Lee CH, Wessling-Resnick M. 010Inflammation-induced iron transport and metabolism by brain microglia. J Biol Chem (2018) 293(20):7853-63. doi: 10.1074/ jbc.RA118.001949

81. Drummond RA. Neuro-Immune Mechanisms of Anti-Cryptococcal Protection. J Fungi (Basel Switzerland) (2017) 4(1):4. doi: 10.3390/ jof 4010004

82. Blasi E, Barluzzi R, Mazzolla R, Tancini B, Saleppico S, Puliti M, et al. Role of nitric oxide and melanogenesis in the accomplishment of anticryptococcal activity by the BV-2 microglial cell line. J Neuroimmunol (1995) 58(1):111-6. doi: 10.1016/0165-5728(95)00016-U

83. Saleppico S, Boelaert JR, Omodeo Salè F, Mazzolla R, Morucci P, Bistoni F, et al. Differential effects of iron load on basal and interferon-gamma plus lipopolysaccharide enhance anticryptococcal activity by the murine microglial cell line BV-2. J Neuroimmunol (1999) 93(1-2):102-7. doi: 10.1016/S0165-5728(98)00206-9

84. D'autréaux B, Toledano MB. ROS as signalling molecules: mechanisms that generate specificity in ROS homeostasis. Nature reviews. Mol Cell Biol (2007) 8(10):813-24. doi: 10.1038/nrm2256

85. Spooner R, Yilmaz O. The role of reactive-oxygen-species in microbial persistence and inflammation. Int J Mol Sci (2011) 12(1):334-52. doi: 10.3390/ijms12010334

86. Brieger K, Schiavone S, Miller FJ, Krause KH. Reactive oxygen species: from health to disease. Swiss Med Weekly (2012) 142:w13659. doi: 10.4414/ smw.2012.13659

87. Zhang Y, Dawson VL, Dawson TM. Oxidative stress and genetics in the pathogenesis of Parkinson's disease. Neurobiol Dis (2000) 7(4):240-50. doi: 10.1006/nbdi.2000.0319

88. Qin B, Cartier L, Dubois-Dauphin M, Li B, Serrander L, Krause K-H. A key role for the microglial NADPH oxidase in APP-dependent killing of neurons. Neurobiol Aging (2006) 27(11):1577-87. doi: 10.1016/ j.neurobiolaging.2005.09.036

89. Krause K-H. Aging: a revisited theory based on free radicals generated by NOX family NADPH oxidases. Exp Gerontol (2007) 42(4):256-62. doi: 10.1016/j.exger.2006.10.011

90. Lopez O, Bonnefont-Rousselot D, Mollereau M, Olivier R, Montagnier L, Emerit J, et al. 003Increased plasma thiobarbituric acid-reactive substances (TBARS) before opportunistic infection symptoms in HIV infected individuals. Clin Chim Acta (1996) 247(1-2):181-7. doi: 10.1016/00098981(95)06249-1

91. Masliah E, Ge N, Mucke L. 004Pathogenesis of HIV-1 associated neurodegeneration. Crit Rev Neurobiol (1996) 10(1):57-67. doi: 10.1615/ CritRevNeurobiol.v10.i1.30

92. Adibhatla RM, Hatcher JF. 005Lipid oxidation and peroxidation in CNS health and disease: from molecular mechanisms to therapeutic opportunities. Antioxid Redox Signal (2010) 12(1):125-69. doi: 10.1089/ ars.2009.2668

93. Keller JN, Mattson MP. 006Roles of lipid peroxidation in modulation of cellular signaling pathways, cell dysfunction, and death in the nervous system. Rev Neurosci (1998) 9(2):105-16. doi: 10.1515/REVNEURO.1998.9.2.105

94. Braughler JM, Hall ED. 008Central nervous system trauma and stroke. I. Biochemical considerations for oxygen radical formation and lipid peroxidation. Free Radic Biol Med (1989) 6(3):289-301. doi: 10.1016/08915849(89)90056-7 
95. Butterfield DA. 007Brain lipid peroxidation and alzheimer disease: Synergy between the Butterfield and Mattson laboratories. Ageing Res Rev (2020) 64:101049. doi: 10.1016/j.arr.2020.101049

96. Dai Y-N, Huang H-J, Song W-Y, Tong Y-X, Yang D-H, Wang M-S, et al. Identification of potential metabolic biomarkers of cerebrospinal fluids that differentiate tuberculous meningitis from other types of meningitis by a metabolomics study. Oncotarget (2017) 8(59):100095-112. doi: 10.18632/ oncotarget.21942

97. Liew KL, Jee JM, Yap I, Yong PVC. In Vitro Analysis of Metabolites Secreted during Infection of Lung Epithelial Cells by Cryptococcus neoformans. PLoS One (2016) 11(4):e0153356. doi: 10.1371/journal.pone.0153356

98. Lau A, Tymianski M. 004Glutamate receptors, neurotoxicity and neurodegeneration. Pflugers Arch (2010) 460(2):525-42. doi: 10.1007/ s00424-010-0809-1

99. Ostrosky-Zeichner L, Soto-Hernandez JL, Angeles-Morales V, Teixeira F, NavaRuiz C, Rios C, et al. 003Effects of pentoxifylline or dexamethasone in combination with amphotericin $\mathrm{B}$ in experimental murine cerebral cryptococcosis: evidence of neuroexcitatory pathogenic mechanisms. Antimicrob Agents Chemother (1996) 40(5):1194-7. doi: 10.1128/AAC.40.5.1194

100. Linkermann A, Stockwell BR, Krautwald S, Anders HJ. Regulated cell death and inflammation: an auto-amplification loop causes organ failure. Nat Rev Immunol (2014) 14(11):759-67. doi: 10.1038/nri3743

101. Martin-Sanchez D, Ruiz-Andres O, Poveda J, Carrasco S, Cannata-Ortiz P, Sanchez-Niño MD, et al. Ferroptosis, but Not Necroptosis, Is Important in Nephrotoxic Folic Acid-Induced AKI. J Am Soc Nephrol (2017) 28(1):21829. doi: 10.1681/ASN.2015121376

102. Linkermann A, Skouta R, Himmerkus N, Mulay SR, Dewitz C, De Zen F, et al. Synchronized renal tubular cell death involves ferroptosis. Proc Natl Acad Sci U S A (2014) 111(47):16836-41. doi: 10.1073/pnas.1415518111

103. Li W, Feng G, Gauthier JM, Lokshina I, Higashikubo R, Evans S, et al. Ferroptotic cell death and TLR4/Trif signaling initiate neutrophil recruitment after heart transplantation. J Clin Invest (2019) 129(6):2293304. doi: $10.1172 /$ JCI126428

104. Funk CD. Prostaglandins and leukotrienes: advances in eicosanoid biology. Science (2001) 294(5548):1871-5. doi: 10.1126/science.294.5548.1871

105. Li C, Deng X, Zhang W, Xie X, Conrad M, Liu Y, et al. Novel Allosteric Activators for Ferroptosis Regulator Glutathione Peroxidase 4. J Med Chem (2019) 62(1):266-75. doi: 10.1021/acs.jmedchem.8b00315

106. Dennis EA, Norris PC. Eicosanoid storm in infection and inflammation. Nat Rev Immunol (2015) 15(8):511-23. doi: 10.1038/nri3859

107. Tobin DM, Vary JC Jr., Ray JP, Walsh GS, Dunstan SJ, Bang ND, et al. The lta4h locus modulates susceptibility to mycobacterial infection in zebrafish and humans. Cell (2010) 140(5):717-30. doi: 10.1016/j.cell.2010.02.013

108. Coulombe F, Jaworska J, Verway M, Tzelepis F, Massoud A, Gillard J, et al. Targeted prostaglandin E2 inhibition enhances antiviral immunity through induction of type I interferon and apoptosis in macrophages. Immunity (2014) 40(4):554-68. doi: 10.1016/j.immuni.2014.02.013

109. Huang JT, Welch JS, Ricote M, Binder CJ, Willson TM, Kelly C, et al. Interleukin4-dependent production of PPAR-gamma ligands in macrophages by $12 / 15$ lipoxygenase. Nature (1999) 400(6742):378-82. doi: 10.1038/22572

110. Murakami K, Ide T, Suzuki M, Mochizuki T, Kadowaki T. Evidence for direct binding of fatty acids and eicosanoids to human peroxisome proliferators- activated receptor alpha. Biochem Biophys Res Commun (1999) 260(3):60913. doi: 10.1006/bbrc.1999.0951

111. Wen Q, Liu J, Kang R, Zhou B, Tang D. The release and activity of HMGB1 in ferroptosis. Biochem Biophys Res Commun (2019) 510(2):278-83. doi: 10.1016/j.bbrc.2019.01.090

112. Kang R, Chen R, Zhang Q, Hou W, Wu S, Cao L, et al. HMGB1 in health and disease. Mol Aspects Med (2014) 40:1-116. doi: 10.1016/j.mam.2014.05.001

113. Yamazaki T, Hannani D, Poirier-Colame V, Ladoire S, Locher C, Sistigu A, et al. Defective immunogenic cell death of HMGB1-deficient tumors: compensatory therapy with TLR4 agonists. Cell Death Differ (2014) 21 (1):69-78. doi: $10.1038 / \mathrm{cdd} .2013 .72$

114. Wang W, Green M, Choi JE, Gijón M, Kennedy PD, Johnson JK, et al. CD8 (+) $\mathrm{T}$ cells regulate tumour ferroptosis during cancer immunotherapy. Nature (2019) 569(7755):270-4. doi: 10.1038/s41586-019-1170-y

115. Vu K, Eigenheer RA, Phinney BS, Gelli A. Cryptococcus neoformans promotes its transmigration into the central nervous system by inducing molecular and cellular changes in brain endothelial cells. Infect Immun (2013) 81(9):3139-47. doi: 10.1128/IAI.00554-13

116. Horwath MC, Bell-Horwath TR, Lescano V, Krishnan K, Merino EJ, Deepe GS Jr. Antifungal Activity of the Lipophilic Antioxidant Ferrostatin-1. Chembiochem (2017) 18(20):2069-78. doi: 10.1002/cbic.201700105

117. Caseys C. Ferroptosis: A Companion of ROS in Fighting Magnaporthe in Rice. Plant Cell (2019) 31(1):13-4. doi: 10.1105/tpc.18.00970

118. Dangol S, Chen Y, Hwang BK, Jwa NS. Iron- and Reactive Oxygen SpeciesDependent Ferroptotic Cell Death in Rice-Magnaporthe oryzae Interactions. Plant Cell (2019) 31(1):189-209. doi: 10.1105/tpc.18.00535

119. Sun Y, Li M, Wang Y, Li L, Wang M, Li X, et al. Ceratocystis fimbriata Employs a Unique Infection Strategy Targeting Peltate Glandular Trichomes of Sweetpotato (Ipomoea batatas) Plants. Phytopathology (2020) 110 (12):1923-33. doi: 10.1094/PHYTO-05-20-0165-R

120. O'rourke JM, Sagar VM, Shah T, Shetty S. Carcinogenesis on the background of liver fibrosis: Implications for the management of hepatocellular cancer. World J Gastroenterol (2018) 24(39):4436-47. doi: 10.3748/wjg.v24.i39.4436

121. Kuo CY, Chiu V, Hsieh PC, Huang CY, Huang SJ, Tzeng IS, et al. Chrysophanol attenuates hepatitis $\mathrm{B}$ virus $\mathrm{X}$ protein-induced hepatic stellate cell fibrosis by regulating endoplasmic reticulum stress and ferroptosis. J Pharmacol Sci (2020) 144(3):172-82. doi: 10.1016/ j.jphs.2020.07.014

122. Kang R, Zeng L, Zhu S, Xie Y, Liu J, Wen Q, et al. Lipid Peroxidation Drives Gasdermin D-Mediated Pyroptosis in Lethal Polymicrobial Sepsis. Cell Host Microbe (2018) 24(1):97-108.e4. doi: 10.1016/j.chom.2018.05.009

Conflict of Interest: The authors declare that the research was conducted in the absence of any commercial or financial relationships that could be construed as a potential conflict of interest.

Copyright (c) $2021 \mathrm{Xu}, \mathrm{Lin}, \mathrm{Tu}, \mathrm{Gao}$, Shao and Sheng. This is an open-access article distributed under the terms of the Creative Commons Attribution License (CC BY). The use, distribution or reproduction in other forums is permitted, provided the original author(s) and the copyright owner(s) are credited and that the original publication in this journal is cited, in accordance with accepted academic practice. No use, distribution or reproduction is permitted which does not comply with these terms. 